\title{
A Mixed Approach for Modeling and Fault Diagnosis of Hybrid Dynamical Systems with Unknown Disturbances
}

\author{
Bochra MAAREF ${ }^{1 *}$, Jalel GHABI ${ }^{1}$, Zineb SIMEU-ABAZI ${ }^{2}$ \\ ${ }^{1}$ LARATSI, National Engineering School of Monastir, Tunisia \\ bochra.maaref.87@gmail.com (*Corresponding author),jalel.ghabi@gmail.com \\ ${ }^{2}$ Grenoble-INP / UJF-Grenoble 1 / CNRS, G-SCOP UMR5272 Grenoble, F-38031, France \\ zineb.simeu-abazi@grenoble-inp.fr
}

\begin{abstract}
A hybrid dynamical system (HDS) results from the interaction between continuous and discrete dynamics. The continuous dynamics represents the system behavior which may change from a functioning mode to another depending on the discrete dynamics. The HDS is often subject to disturbances arising from external phenomena due to the environment, or internal phenomena linked to changes in the system. This paper presents a diagnosis approach of disturbed complex HDS by combining State Observers and Timed Automata (TA). The proposed method combines the advantages of observer and TA to get the best performance in the presence of disturbances, particularly in the fault location phase. Thus, for complex systems, the observer-based approach is used to generate fault indicators for fault detection and the fault location procedure is ensured by the signature matrix. The TA, which are commonly used in discrete event systems enables to take into account temporal aspects and to follow up the system dynamic evolution as well as fault propagation effects. To refine fault location, a TAbased diagnoser is constructed. The simulation results show the dynamic behavior of the system variables with performance evaluation of the proposed fault diagnosis approach.
\end{abstract}

Keywords: Hybrid Dynamic Systems, Observer, Timed Automata, Fault diagnosis, Disturbances.

\section{Introduction}

Dependability can be invoked when faults occur in the system. This observation obviously leads to the execution of a monitoring system able, at all times, to provide the operating state of the components constituting a physical system (actuator, sensor, etc.). The first objective of the monitoring function is to increase the safety of the installation. In this context, many approaches have been developed by the different research communities, with a view to fault detection and diagnosis. Methods differ with respect to the type of prior knowledge on the processes that they require. Thus, they are classified in two classes depending upon being based on a model or not. Methods of supervising without model are based on information obtained from previous experience, on heuristic rules or on examples of resolution. For instance, an expert in a field uses the experience he has acquired when he is confronted the similar cases of supervision. He can also exploit physical laws describing the expected behavior of the system. Any deviation from the expected "normal" behavior is considered a symptom of a failure. The fundamental principle of this approach, which groups the methods of supervising with analytical models, consists in comparing the consistency of the simulated model with the observations of the real device. Some methods without model are given in literature such as the Artificial Neural Networks (ANN)
[15], the Fuzzy Inference Systems (FIS) [1] and the Recognition Of Shapes (ROS) [3]. Modelbased monitoring methods have been developed since the early 1970s [8]. They are based on the generation and the study of a particular signal called "fault indicator" or "residual" [7]. The models used can be: continuous time or discrete time, qualitative, structural or analytical, linear or not, representing the proper functioning or taking into account the failures. We focus then, in this paper, on the method based on models to monitor the Hybrid Dynamic Systems (HDS) [11] composed of discrete and continuous dynamics which interact. The discrete part is described by state transitions and the continuous by differential equations. However, a physical system is often subject to perturbations which can be assimilated to unknown input and which have negative effects on the system behavior. The objective of this paper is the modeling and the diagnosis of HDSs subjected to perturbations. For the modeling we adapt a mixed approach which combines the continuous system approach and the Discrete Event System (DES) one. For diagnosis, we use the residue generation technique for the continuous systems and the timed model for the monitoring discrete systems. Two modeling and analysis tools are coupled: The State Observer and the Timed Automata (TA). Compared to hybrid 
automata our diagnosis procedure takes into account the system perturbations and enables to locate all the faults.

This paper is structured as follows: Section 2 rapidly outlines our problem. The generic structure of HDSs and the so-called mixed modeling approach are detailed in Section 3. Our proposed approach concerned with fault diagnosis in the presence of disturbances or noise is presented in Section 4. Then, Section 5 presents the case study using a four-tank system and illustrates the efficiency of the proposed approach through simulation results in Section 6. Finally, section 7 concludes the paper.

\section{Problem Statement}

A Hybrid Dynamic System is often subject to disturbances which originate from external phenomena due to the environment, or internal phenomena related to system changes. These disturbances which can be assimilated to unknown inputs have negative effects on the system behavior. The main objective of the paper is to propose a new approach for the fault detection and the possibility of distinguishing the component defect from the defect due to disturbance in a Hybrid Dynamic System.

\section{Modeling of Hybrid Dynamical Systems}

The industrial processes are complex; their dynamics present a double aspect in other words, a continuous and/or discrete nature.

The structural changes in these systems lead to discontinuities. They may occur because of discrete events designed by discrete sensors, actuators, or may be inherent in the system. However, in order to ensure the proper functioning of the whole automated system, it is essential to take into account the continuous as well as the discrete aspects of its dynamics. This problematic which is interested in continuous and discrete phenomena in a global sense is relatively recent (90s). It generates a very important and very particular class of systems known as the Hybrid Dynamic System (HDS). For the modeling of HDSs, there are several approaches which share the idea that continuous system evolution is affected by the discrete events. Therefore, the HDS could not be handled with classical approaches based on homogeneous modeling. Thus the complex systems are designed by incorporating differential equations due to model continuous behavior and discrete event representations due to instantaneous change of state in response to events. These systems have some signals or variables that take their values in a continuous set and other variables that take values in a finite discrete set. Therefore, the system behavior is determined by the interaction between discrete and continuous dynamics. A hybrid system will be then modeled by a set of continuous dynamics corresponding to different discrete events. Mainly, there are three modeling approaches of HDSs: the continuous, the event and the mixed approaches. The first consists in defining an approximation of the discrete dynamics by differential equations to model the occurrence of discrete events so that the HDS will behave as a continuous system. This representation enables us to use the continuous system properties for HDS. The second approach is purely discrete. It consists in approximating the continuous evolution so that the hybrid system is represented only by the events that characterize it which will allow to use the classical theory of supervisor of the DESs for control synthesis. The third is the mixed approach which is based on the assumption that the operation of a hybrid system is carried out in two steps. The first corresponds to a transformation of the continuous state described in terms of time elapsed during this phase and the second considers the instantaneous discrete change of this state. The models developed in this approach are based on the interaction of two sub-models, one for the event aspects, based on finite state automata, Petri nets or extensions of these formalisms, and the other, formalized by equations of state (often by differential equations) for continuous aspects. Each of the aspects is clearly considered in the interface between both sub-models. The event aspect influences the continuous model by validating some of the continuous equations as a function of the discrete active state and the continuous aspect acts on the event model by validating or forcing the crossing of certain transitions. Among the modeling tools resulting from this mixed approach, we find the hybrid automata [2] representing the fundamental formal model of this approach, the hybrid statecharts to solve the problems posed by model specification, particularly in the hierarchical 
structuring [6], and finally the different extensions of the Petri Nets [5]. The Figure 1-a gives the principle of this model where the continuous part is modelled by differential equations (Simulink) and discrete part by automaton (Stateflow) [10]. The temporal evolution is illustrated by the curves in Figure 1-b.

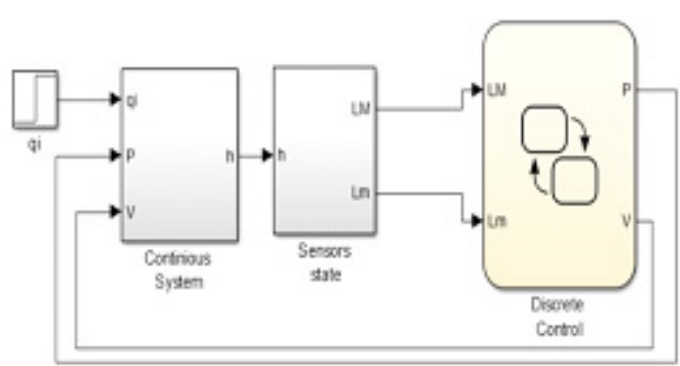

-

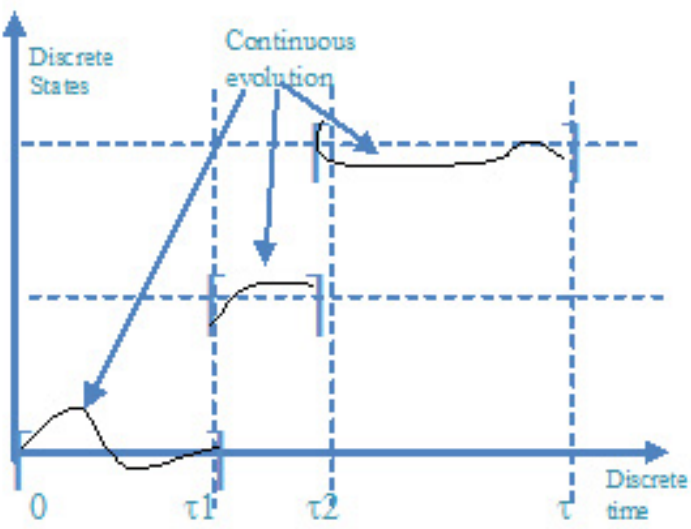

$-b-$

Figure 1 - a) Hybrid systems modeling; b) time evolution of hybrid systems

The mixed approach in Figure 2 represents a finite state automata which controls differential equations. In fact, the model is represented by the set $X$ of real variables and by the set $(S, E)$ event graph. The set $S$ consists of the vertices of the graph and so the set $E$ represents the discrete transitions.

\section{Monitoring Hybrid Dynamical Systems}

When the process is described by a HDS, few works has been proposed for detecting or locating failures. Using residuals, numerous solutions have been suggested for continuous and discrete systems, linear and nonlinear [14] [13]. For the mixed approach proposed in this paper, the diagnosis approach combines the advantages of both methods (Observer and TA) to achieve the best performance, particularly in the fault location phase.

\subsection{Diagnosis of the Continuous Part}

In the continuous part, the first step is to generate residues that are sensitive to certain failures and robust to disturbances and other failures. The residuals describe the differences in the responses between the system behaviors and the model. The algorithm used to obtain the residues is called the residue generator. Three approaches are mainly used to construct this type of residue: the Identification Approaches [13], the Analytical Redundancy Relations (ARR) or Parity Space approach [4] and the observer-based approaches or filters [14]. In this paper, we are interested in the state observer-based approach.

\section{- Diagnosis based on state observer}

The diagnosis based observer compares the estimated output functions with the same functions of the measured outputs to generate a residue or indicator of the failure occurrence which is often modeled by unknown additive

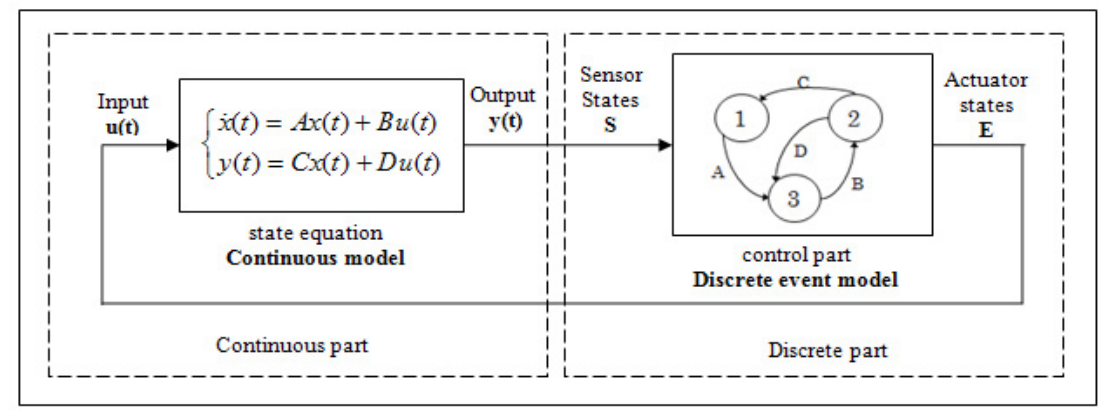

Figure 2. Modeling of HDS through the Mixed Approach 
signals that are of two classes. The first class concerns the signals generated by the occurrence of a fault or a failure of a component (sensor, actuator). In order to clearly highlight these faults, it is necessary to make the output estimation error particularly sensitive to these unmeasured inputs, so that the residue deviates visibly from zero. The second class interests the non-significant signals to which the estimation error must be less sensitive, essentially modeling errors and measurement noises.

A limit of the influence of this class of signals (sensor accuracy, signal-to-noise ratio, etc.) gives a threshold beyond which the deviation between the outputs and their estimates is indicative of a failure. Once the fault is detected we are interested in identifying the component in fault.

The system can be described by the state equations:

$$
\left\{\begin{array}{l}
\dot{x}(t)=A x(t)+B u(t) \\
y(t)=C x(t) \\
x(0)=x_{0}
\end{array}\right.
$$

Where $x(t) \in R^{n}$ is the state vector, $u(t) \in R^{p}$ the input vector and $y(t) \in R^{m}$ is the output vector and the matrices $A, B$ and $C$ are of appropriate dimensions.

In order to diagnose a defect, we define the following observer:

$$
\left\{\begin{array}{l}
\tilde{\dot{x}}(t)=A \tilde{x}(t)+B u(t)+K(y(t)-\tilde{y}(t)) \\
\tilde{y}(t)=C \tilde{x}(t) \\
\tilde{x}(0)=\tilde{x}_{0}
\end{array}\right.
$$

Where $\tilde{x}$ and $\tilde{y}$ are the estimates of $\mathrm{x}$ and $\mathrm{y}$ respectively. Assuming that the pair $(A, C)$ is observable, the observer gain matrix $K$ can be chosen such that $(A-K C)$ is a stable matrix.

\section{- Diagnosis based on unknown input state observer}

Assuming that all the unknown signals acting on the system are considered unknown inputs $w(t) \in R^{q}$, we have:

$\left\{\begin{array}{l}\dot{x}(t)=A x(t)+B u(t)+F w(t) \\ y(t)=C x(t) \\ x(0)=x_{0}\end{array}\right.$

The matrix $F$ is known, constant and with appropriate dimension.
The aim of estimation in the existence of unknown inputs is to determine an observer which produces an estimate $\tilde{x}(t)$ so that the state estimation error $\hat{x}(t)=x(t)-\tilde{x}(t)$ tends asymptotically towards 0 when $t \rightarrow \infty$. The structure of such observer is as follows:

$\left\{\begin{array}{l}\dot{z}(t)=N z(t)+M u(t)+L y(t) \\ \tilde{x}(t)=z(t)+E y(t) \\ z(0)=z_{0}\end{array}\right.$

where $L, M, N$ and $E$ are the observer gains calculated so that $\hat{x}(t)$ converges to zero for any initial state. To ensure the convergence of $\hat{x}(t)$, the following conditions should be satisfied [9]:

$0=P A-L C-N P$
$0=P B-M$
$0=P F$

\section{$N$ is stable}

where the matrix $P$ is defined by:

$P=I_{n}-E C$

From equations (6) and (8) we obtain

$M=\left(I_{n}-E C\right) B$

Substituting (8) into (7), we find:

$E C F=F$

The general solution of (10) can be written as [9]

$E=F(C F)^{+}+\Theta\left(I_{m}-C F(C F)^{+}\right)$

where $\Theta$ is an arbitrary matrix, $I_{\mathrm{x}}$ is the identity matrix of dimension $x$ and $X^{+}$denotes the pseudoinverse of the full column rank matrix $X$, defined as: $X^{+}=\left(X^{\mathrm{T}} X\right)^{-1} X^{\mathrm{T}}$

By posing the matrix $L$ of system (4) as:

$L=K+N E$

From equation (8), (5), (12) and (11) we have:

$N=\left(I_{n}-\left(F(C F)^{+} C\right) A\right.$

$-[\Theta K]\left[\begin{array}{c}\left(I_{m}-C F(C F)^{+}\right) C A \\ C\end{array}\right]$

which determine the observer gains, it is first necessary to determine the matrices $\Theta$ and $K$ of (13) by placing the poles of $N$ in order to guarantee its stability. Then the other gains $M, E$ and $L$ are given by (9), (11) and (12). 


\section{- Residues generation}

Our goal is to construct residual generators $r(t)$ directly from the observer:

$$
r(t)=y(t)-\tilde{y}(t)=C(x(t)-\tilde{x}(t))
$$

Indeed, in case of an observer, the residuals $r(t)$ represent the estimation error. When the system is affected by actuator faults $f_{a}(t)$, sensor faults $f_{s}(t)$ and unknown inputs $w(t)$, it can be modeled by:

$\left\{\begin{array}{l}\dot{x}(t)=A x(t)+B u(t)+F w(t)+D_{a} f_{a}(t) \\ y(t)=C x(t)+D_{s} f_{s}(t) \\ x(0)=x_{0}\end{array}\right.$

The matrices $D_{a}$ and $D_{s}$ are called the fault distribution matrices. The $i^{\text {th }}$ column of $D_{a}$ and the $i^{\text {th }}$ column of $D_{s}$ give the direction of the faults $i$ in the measurement equation.

The estimation error (14), synthesized according to (9), (11), (12) and (13), can be seen as the output of the following dynamic system:

$$
\left\{\begin{array}{l}
\dot{\hat{x}}(t)=N \hat{x}(t)+P D_{a} f_{a}(t) \\
+(N E-L) D_{s} f_{s}(t)-E D_{s} \dot{f}_{s}(t) \\
r(t)=\hat{y}(t)=C \hat{x}(t)+D_{s} f_{s}(t) \\
\hat{x}(0)=P x_{0}-z_{0}
\end{array}\right.
$$

It is obvious that in the absence of a defect the signal $\hat{y}(t)=y(t)-\tilde{y}(t)$ converges asymptotically to zero. The signal $\hat{y}(t)$ is therefore a residue which is sensitive to $f_{a}(t)$ and $f_{s}(t)$ but insensitive to $w(t)$. The detection step is very important in system diagnosis. It involves the evaluation of residues which are directly related to the variations coming from the outputs (measurement noise, sensor defects) and inputs (actuator faults, disturbances, etc.). Therefore, an alarm $A_{i}(t)$ assigned to the $i^{\text {th }}$ defect is obtained by comparing $\hat{y}_{i}(t)$ (or residue $\left.r_{i}(t)\right)$ with a given threshold $\varepsilon_{i}$.

$$
A_{i}(t)=\left\{\begin{array}{lll}
0, & \text { if } & \left|\hat{y}_{i}(t)\right|<\varepsilon_{i} \\
1, & \text { if } & \left|\hat{y}_{i}(t)\right| \geq \varepsilon_{i}
\end{array}\right.
$$

The fault location results from the signature matrix. The residuals are designed to be affected each one by a sub-set of faults and insensitive to the remaining faults. Thus, only a subset of residuals reacts, when a fault appears. Thereafter, the signature matrix gathers the sensitivity information for the residuals. It is defined as follows:
$M_{R}: R \times F \mapsto\{0,1\}$

$(r, f) \rightarrow M_{R}(i, j)$

Where $M_{R}(i, j)$ is the $(i, j)^{\text {th }}$ component of the signature matrix $M_{R}$, such that

$M_{R}(i, j)=\left\{\begin{array}{l}1 \text { if and only if } r \text { is sensitive to the defect } f \\ 0 \text { if and only if } r \text { is insensitive to the defect } f\end{array}\right.$

$M_{R}=\left(\begin{array}{cccc}1 & 0 & \cdots & 0 \\ 0 & 1 & \ddots & \vdots \\ \vdots & \ddots & \ddots & 0 \\ 0 & \cdots & 0 & 1\end{array}\right)$

The dimensions of the signature matrix are determined from the number of sensors or actuators and the number of residues generated by the observer model. The $j^{\text {th }}$ column of $M_{R}$ corresponds to the residue $r_{j}$ and the $i^{\text {th }}$ line corresponds to the defect $f_{i}$.

\subsection{Diagnosis of the Discrete Part}

The DESs are described either by models where time is deterministic (temporal Petri nets, TA) or by models where time is random (Markov chain).

\section{- Diagnosis by timed automata}

Our objective is to design a diagnoser, which allows analyzing, detecting and locating a fault in a system. The method used is based on the employment of TA which is a tool for modeling and monitoring real-time systems [12]. It is an extension by real variables, called clocks, of the finite state machines. Thus a TA can be considered as an abstract model of a timed system. A TA is defined by a 7- uplet $A=\left(Q, X, \Sigma, q_{F}, q_{0}, I, T\right)$; Where $Q=\{q i: i=0,1,2, \ldots\}$ is a finite set of states, $\mathrm{X}=\{\mathrm{x} 1, \ldots, \mathrm{xn}\}$ is a finite set of variables (clocks), $\Sigma$ is a finite set of events, $\mathrm{q}_{\mathrm{F}} \subseteq \mathrm{Q}$ is the set of final states, $\mathrm{q}_{0} \in \mathrm{Q}$ is an initial state, $\mathrm{I}: \mathrm{Q} \rightarrow$ $\mathrm{C}(\mathrm{X})$ is a function which associates to each state a temporal constraint, called an invariant, $\mathrm{T} \subset \mathrm{Q}$ $\times \Sigma \times \mathrm{C}(\mathrm{X}) \times 2^{\mathrm{X}}$ is a set of action transitions. In Figure 3 we illustrate a diagnoser, using TA, designed to diagnose a fault in a process. Diagnoser constructor is thus based on a model representing two modes: the normal mode and the defective mode. The dynamical model, as seen in Figure 3-(a), is a control-command program of the process to which time information is added, for example the order of task execution, the date of event occurrence and the duration of the various functioning steps. The role of the diagnoser is to deduce the existence of unobservable faults based on observable events and the time elapsed between them. 


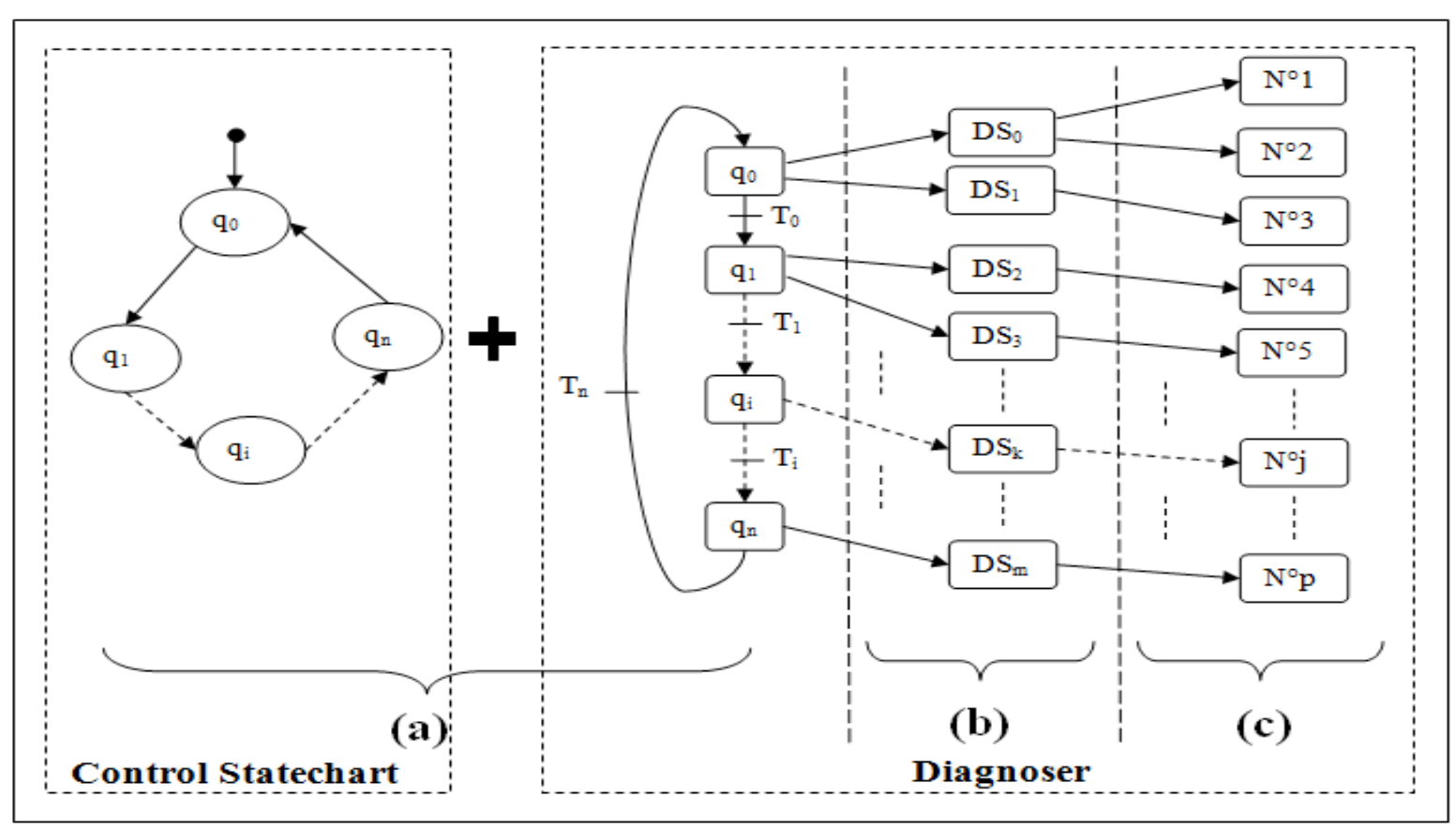

Figure 3. General Construction of a Diagnoser

a) Dynamical model, b) Detection stage, c) Location stage DS: Detection Step; $\mathrm{N}^{\circ} \mathrm{j}$ : Fault $\mathrm{N}^{\circ} \mathrm{j}$.

\section{- Principle of fault detection}

The temporal evolution of the process results from a succession of states (Figure 3- (b)). A discrete transition $T i$ from state $i$ to the state $i+1$ occurs when two conditions are satisfied. The first is related to certain logical conditions which can be provoked by discrete events caused by discrete sensors or actuators. The second is related to given time $T a$ that should be elapsed. This time is within an interval $\left[T_{\min }, T_{\max }\right]$ in normal mode and ] Tmax, $T c]$ in degraded mode. When $T a$ is greater than $T c$ we assist to a faulty mode (see Figure 4).

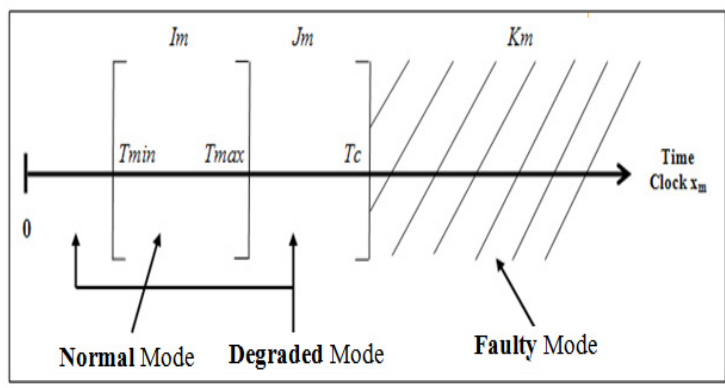

Figure 4. Functioning Modes

\section{- Fault detection condition}

The state evolution is accompanied by the computing of the residual $r(t)$ given by (16). Three situations can occur:

- If $r(t)=0$ when $t \in\left[T_{\min }, T_{\max }\right]$, the system is in normal mode.
- If $r(t)=0$ when $t \in] T_{\text {max }}, T_{C}[$, the system is a degraded mode

- If $r(t) \neq 0$ when $t \in] T_{C}$, $+\infty[$, the system is a faulty mode.

The advantage of this condition is to identify the degraded mode of the system without calculating the critical time $T c$ which is difficult to determine. Figure 4 illustrates the three functioning intervals.

\section{- Principle of fault location}

The second step of the diagnosis concerns the fault location. Following the fault detection and the different faulty modes identified in the FMEA (Failure Modes and Effects Analysis), we determined the faulty component or a set of defective components, Figure 3-c. The dynamic model of the process contains two states: fault state and normal state. From there, through the path from the initial state to the fault state, the fault is located by measuring the elapsed times in the different transitions.

The steps of the algorithm which summarizes our diagnostic approach are:

1: Model a HDS subject to disturbances by connecting two tools:

1.1: The unknown input observer concerns 
the continuous part of the HDS.

1.2: The timed automata handles the discrete part.

2: Diagnose a fault by our model:

2.1: Detect a fault:

2.1.1: Analyze the residuals.

2.1.2: Establish the dynamics of the hybrid system to determine characteristic times.

2.1.3: Apply the fault detection condition to distinguish the fault of a system component and the fault resulting from the disturbance.

2.2: Locate a fault by analyzing the signature matrix and by temporal identification of the system in order to locate all the defects.

\section{Application: Water Distribution Network}

\subsection{System Description}

The process of water distribution network is shown in Figure 5. This network is made up of four tanks each with a ground section $S_{i}, i=1, \ldots 4$, connected by the same pipes with the same section $s_{p}$, equipped with the valves $V_{1}, V_{2}$ and the valve $V_{3}$ which allows the liquid evacuation. The network is supplied with two volumetric flows $q_{e l}(t)$ and $q_{e 2}(t)$ feeding the tanks 1 and 2 respectively and controlled by pumps $P_{1}$ and $P_{2}$. To observe the network, there are three flow meters $Q_{1}, Q_{2}$ and $Q_{3}$ marked in Figure 5 by black circles. The sensors are calibrated to measure the variation around steady state values. The available measurements are the sum of the outflows of the tanks 1 and 2 (through valve $\left.V_{1}\right): y_{1}(t)=q_{1}(t)+q_{2}(t)$, the outflow of the tank 3 through valves $V_{2}, y_{2}(t)=q_{3}(t)$ and that of tank 4 through $V_{3}, y_{3}(t)=q_{4}(t)$. The water levels in the tanks 1 and 2 are obtained through four sensors: two for high levels $L_{l h}$ and $L_{2 h}$ and two for low levels $L_{1 b}$ and $L_{2 b}$. The controls of the valves $V_{1}, V_{2}, V_{3}$ and the pumps $P_{1}, P_{2}$ are on/off controls. When $P_{1}$ is stopped its flow $q e_{1}(t)=0$ and when it is running $q e_{1}(t)=0,02 \mathrm{~m}^{3} / \mathrm{s}$, similarly for $P_{2}$. The discrete event $V_{1}, V_{2}, V_{3}, P_{1}, P_{2}, L_{1 h}$, $L_{2 h}, L_{1 b}, L_{2 b}$ are Boolean variables. All these events are numerically controlled except $V_{1}, V_{2}$ and $V_{3}$ which are manual. The objective is to maintain the liquid levels $h_{1}$ and $h_{2}$ in tanks 1 and 2 within the following interval:

$\left\{\begin{array}{l}1.3 m \leq h_{1} \leq 12 m \\ 0.5 m \leq h_{2} \leq 7 m\end{array}\right.$

Two non-measurable perturbations quantities may be assumed to affect the evolution of the network: a leakage in the supply pipe of the tank 1 denoted $f(t)$ or an infiltration in the tank 4 , denoted $d(t)$.

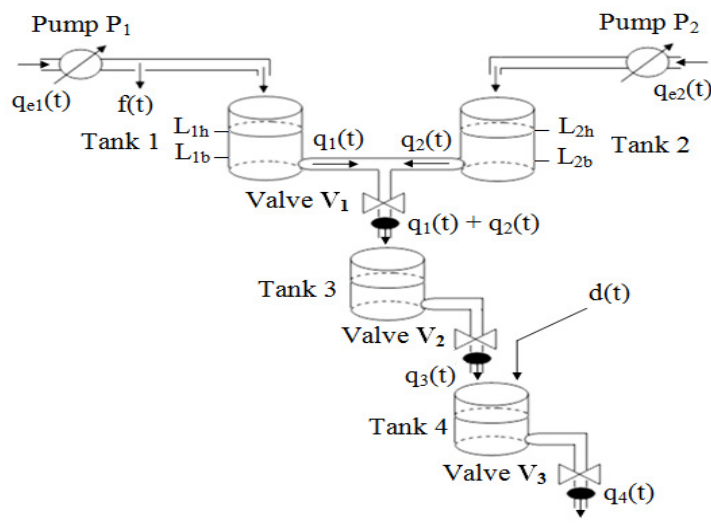

Figure 5 Water distribution network

\subsection{Systems Diagnosis}

\subsubsection{Diagnosis of Failures Influencing Continuous Dynamics}

While using the principle of mass conservation, our system can be represented by the system of differential equations. Using Torricelli's law [9], we have:

$q(t)=s_{p} \operatorname{sign}(\Delta h) \sqrt{2 g|\Delta h|}$

Where $\boldsymbol{q}(\boldsymbol{t})$ is the flow in $\mathrm{m}^{3} / \mathrm{s}, \boldsymbol{s}_{\boldsymbol{p}}$ is the section of the pipe in $m^{2}, \Delta \boldsymbol{h}$ is the level difference between the tanks in $m$ and $\operatorname{sign}(\Delta \boldsymbol{h})$ means the sign of $\Delta h$.

Thus, from relation (21) and Figure 5 we have: $q_{i}(t)=s_{p} \sqrt{2 g h_{i}}$, where $h_{i}$ is the water level in the tank $i, i=1, \ldots 4$.

The system of water distribution network is described by the following four nonlinear differential equations: 


$$
\left\{\begin{array}{l}
S_{1} \frac{d h_{1}}{d t}=q_{e 1}-q_{1} \\
S_{2} \frac{d h_{2}}{d t}=q_{e 2}-q_{2} \\
S_{3} \frac{d h_{3}}{d t}=q_{1}+q_{2}-q_{3} \\
S_{4} \frac{d h_{4}}{d t}=q_{3}-q_{4}
\end{array}\right.
$$

By neglecting the load losses in the pipes and after linearization around the stationary operating regime, the continuous part of the system can be modeled in the following form:

$$
\begin{aligned}
& \left(\begin{array}{l}
\delta \dot{q}_{1}(t) \\
\delta \dot{q}_{2}(t) \\
\delta \dot{q}_{3}(t) \\
\delta \dot{q}_{4}(t)
\end{array}\right)=\left(\begin{array}{cccc}
-a_{1} & 0 & 0 & 0 \\
0 & -a_{2} & 0 & 0 \\
a_{3} & a_{3} & -a_{3} & 0 \\
0 & 0 & a_{4} & -a_{4}
\end{array}\right)\left(\begin{array}{l}
\delta q_{1}(t) V_{1} \\
\delta q_{2}(t) V_{1} \\
\delta q_{3}(t) V_{2} \\
\delta q_{4}(t) V_{3}
\end{array}\right) \\
& +\left(\begin{array}{cc}
a_{1} & 0 \\
0 & a_{2} \\
0 & 0 \\
0 & 0
\end{array}\right)\left(\begin{array}{ll}
\delta q_{e 1}(t) & P_{1} \\
\delta q_{e 2}(t) & P_{2}
\end{array}\right)+\left(\begin{array}{cc}
a_{1} & 0 \\
0 & 0 \\
0 & 0 \\
0 & a_{4}
\end{array}\right)\left(\begin{array}{l}
f(t) \\
d(t)
\end{array}\right) \\
& \delta y(t)=\left(\begin{array}{llll}
1 & 1 & 0 & 0 \\
0 & 0 & 1 & 0 \\
0 & 0 & 0 & 1
\end{array}\right)\left(\begin{array}{l}
\delta q_{1}(t) V_{1} \\
\delta q_{2}(t) V_{1} \\
\delta q_{3}(t) V_{2} \\
\delta q_{4}(t) V_{3}
\end{array}\right)+\left(\begin{array}{l}
v_{1}(t) \\
v_{2}(t) \\
v_{3}(t)
\end{array}\right)
\end{aligned}
$$

with: $P_{1}, P_{2}, V_{1}, V_{2}, V_{3} \in\{0,1\}$

where $\delta x(t)$ refers to the variation of $x(t)$ around its steady state value. The coefficients $a_{i}$ are given by $a_{i}=\frac{s_{p}}{S_{i}} \sqrt{\frac{g}{2 h_{i 0}}}$, Where $h_{i 0}$ is the water level in the tank $i$ in stationary operation. Tank sections are: $S_{1}=100 \mathrm{~m}^{2}, S_{2}=150 \mathrm{~m}^{2}, S_{3}=300 \mathrm{~m}^{2}, S_{4}$ $=400 \mathrm{~m}^{2}$. The stationary regime imposed by feeding rates $q_{e 1}(t)=0,02 \mathrm{~m}^{3} / \mathrm{s}$ and $q_{e 2}(t)=0,015$ $\mathrm{m}^{3} / \mathrm{s}$ is characterized by: $a_{1}=49,05 \times 10^{-4} \mathrm{~s}^{-1}, a_{2}$ $=43,60 \times 10^{-4} s^{-1}, a_{3}=9,3429 \times 10^{-4} s^{-1}$ and $a_{4}=$ $7,0071 \times 10^{-4} s^{-1}$. The measurements are affected by sensor noises, denoted $v_{i}(t)$, with zero mean and standard deviation $\sigma=5 \times 10^{-5}$. To satisfy the conditions of existence of an observer with unknown inputs, the number of measurements should be strictly superior to the number of unknown inputs. In addition, we can verify the observability of (13), that is, the eigenvalues of matrix $\mathrm{N}$ can be arbitrarily placed by means of $\Theta$ and $K$. The poles of the observer are:
$\{-0.0196-0.0206-0.0216-0.0196\}$. The gains $N$, $E, M$ and $L$ are calculated from (9), (11), (12) and (13).

$$
K=\left[\begin{array}{ccc}
0.045 & -0.015 & 0 \\
-0.0099 & 0.0084 & 0 \\
-0.0032 & 0.021 & 0 \\
0 & 0.0007 & 0.020
\end{array}\right] \Theta=\frac{1}{10000}\left[\begin{array}{ccc}
0 & 0.5608 & 0 \\
0 & -0.1711 & 0 \\
0 & -0.2260 & 0 \\
0 & -0.0065 & 0
\end{array}\right]
$$

The leakage rate $f(t)$ is equal to $30 \%$ of $\delta q e_{1}(t)$ and the infiltration $d(t)$ is equal to $0.01 \mathrm{~m}^{3} / \mathrm{s}$. Both perturbation are applied between time instants $t=600 \mathrm{~s}$ and $t=900 \mathrm{~s}$. The model-based failure detection is based on the evolution of the residuals. Therefore we start by generating residuals sensitive to certain failures and robust to disturbances and other failures. Thus, when a fault occurs, only a subset of residues reacts. The desired sensitivity and robustness information for the residuals is listed in a binary matrix, called the signature matrix, as illustrated in Table 1. This is constructed in the following way: when the $j^{\text {th }}$ residue is to be sensitive (resp. robust) to the $i^{\text {th }}$ failure, then the binary value 1 (resp. 0) is assigned to the $(i, j)^{\text {th }}$ component of the matrix. Fault location, in fact, is studied by analyzing the fault signature matrix. However, in the case of multiple signatures, this technique does not allow then the fault location. To do this, we build a diagnoser based on TA which takes into account dynamic evolution of the system and failure propagation.

\subsubsection{Diagnosis of Failures Influencing Discrete Dynamics}

In general, the discrete evolution of a HDS is determined by the occurrence of external or internal events the nature of which may be controllable or not (for instance, the intervention of a human operator or the failure of components). The occurrence of events implies changes in the continuous dynamics of the system. In Hybrid Dynamic Systems (HDS), these changes can be either by a particular structure of the physical process or by discrete inputs / outputs generated by different components of the system. To simplify the study, it is assumed that the valves $V_{1}, V_{2}$ and $V_{3}$ remain constantly open. The pumps $P_{1}$ and $P_{2}$ are controlled in on/off way to maintain the levels 
Table 1. Matrix of fault signatures.

\begin{tabular}{|c|c|c|c|c|}
\hline Failure mode & Components & $\mathbf{r}_{1}$ & $\mathbf{r}_{2}$ & $\mathbf{r}_{3}$ \\
\hline Flow Sensor Failure $Q_{L}$ & Flow sensors $Q_{1}$ & 1 & 0 & 0 \\
\hline Flow Sensor Failure $Q 2$ & Flow sensors $Q_{2}$ & 0 & 1 & 0 \\
\hline Flow Sensor Failure $Q_{3}$ & Flow sensors $Q_{3}$ & 0 & 0 & 1 \\
\hline Leak in tank 1 & Tank 1 & 1 & 0 & 0 \\
\hline Leak in tank 2 & Tank 2 & 1 & 0 & 0 \\
\hline Leak in tank 3 & Tank 3 & 0 & 1 & 0 \\
\hline Leak in tank 4 & Tank 4 & 0 & 0 & 1 \\
\hline Pump $P_{1}$ Stuck_Close & \multirow{2}{*}{$\operatorname{Pump} P_{1}$} & \multirow{2}{*}{0} & \multirow{2}{*}{1} & \multirow{2}{*}{0} \\
\hline Pump $P$, Stuck_Open & & & & \\
\hline Pump $P_{2}$ Stuck_Close & \multirow{2}{*}{ Pump $P_{2}$} & \multirow{2}{*}{0} & \multirow{2}{*}{1} & \multirow{2}{*}{0} \\
\hline Pump $P_{2}$ Stuck_Open & & & & \\
\hline Level sensor $L_{l h}$ Stuck_Up & \multirow{2}{*}{ Level sensor $L_{1 h}$} & \multirow{2}{*}{0} & \multirow{2}{*}{1} & \multirow{2}{*}{0} \\
\hline Level sensor $L_{l h}$ Stuck_Down & & & & \\
\hline Level sensor $L_{1 b}$ Stuck Up & \multirow{2}{*}{ Level sensor $L_{1 \mathrm{~b}}$} & \multirow{2}{*}{0} & \multirow{2}{*}{1} & \multirow{2}{*}{0} \\
\hline Level sensor $L_{l b}$ Stuck_Down & & & & \\
\hline Level sensor $L_{2 h}$ Stuck_Up & \multirow{2}{*}{ Level sensor $L_{2 \mathrm{~h}}$} & \multirow{2}{*}{0} & \multirow{2}{*}{1} & \multirow{2}{*}{0} \\
\hline Level sensor $L_{2 h}$ Stuck_Down & & & & \\
\hline Level sensor $L_{2 b}$ Stuck_Up & \multirow{2}{*}{ Level sensor $L_{2 \mathrm{~b}}$} & \multirow{2}{*}{0} & \multirow{2}{*}{1} & \multirow{2}{*}{0} \\
\hline Level sensor $L_{2 b}$ Stuck_Down & & & & \\
\hline Valve $V_{1}$ Stuck_Close & \multirow{2}{*}{ Valve $V_{1}$} & \multirow{2}{*}{0} & \multirow{2}{*}{1} & \multirow{2}{*}{1} \\
\hline Valve $V_{1}$ Stuck_Open & & & & \\
\hline Valve $V_{2}$ Stuck_Close & \multirow{2}{*}{ Valve $V_{2}$} & \multirow{2}{*}{0} & \multirow{2}{*}{1} & \multirow{2}{*}{1} \\
\hline Valve $V_{2}$ Stuck_Open & & & & \\
\hline Valve $V_{3}$ Stuck_Close & \multirow{2}{*}{ Valve $V_{3}$} & 0 & 1 & 1 \\
\hline Valve $V_{3}$ Stuck_Open & & 0 & 1 & 1 \\
\hline $\begin{array}{l}\boldsymbol{L}_{i} \text { Stuck_Down means the sensor } \\
\text { Stuck_Up means the sensor always } \\
\text { means the valve number } i \text { remain } \\
\text { remains open to a closing request; } \\
\boldsymbol{P}_{\boldsymbol{i}} \text { Stuck_Open means the pump }\end{array}$ & $\begin{array}{l}\text { ys remains in sta } \\
\text { ins in state } 1 \text { (the } \\
\text { d to an opening } \\
\text { ck_Close means } \\
\text { r } i \text { remains runnir }\end{array}$ & $\mathrm{se}$ & & \\
\hline
\end{tabular}

Table 2. Temporal identification of the system.

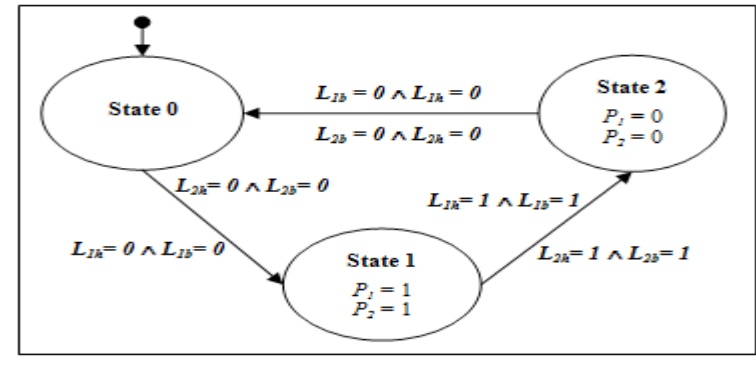

Figure 6. Timed automata of our discrete system (1: pump on; 0: pump off; “ $\wedge ”=$ logical AND).

\begin{tabular}{|c|c|}
\hline Actions & Time in sec \\
\hline$P_{1}$ On & 30 \\
$P_{1}$ Off & 327.16 \\
\hline$P_{2}$ On & 30 \\
$P_{2}$ Off & 378.6 \\
\hline Activating $L_{1 h}$ & 327.16 \\
Deactivating $L_{1 h}$ & 327.16 \\
\hline Activating $L_{2 b}$ & 89.3 \\
Deactivating $L_{2 b}$ & 553.7 \\
\hline Activating $L_{2 h}$ & 378.6 \\
Deactivating $L_{2 h}$ & 378.6 \\
\hline Activating $L_{2 b}$ & 83.7 \\
Deactivating $L_{2 b}$ & 681.26 \\
\hline
\end{tabular}




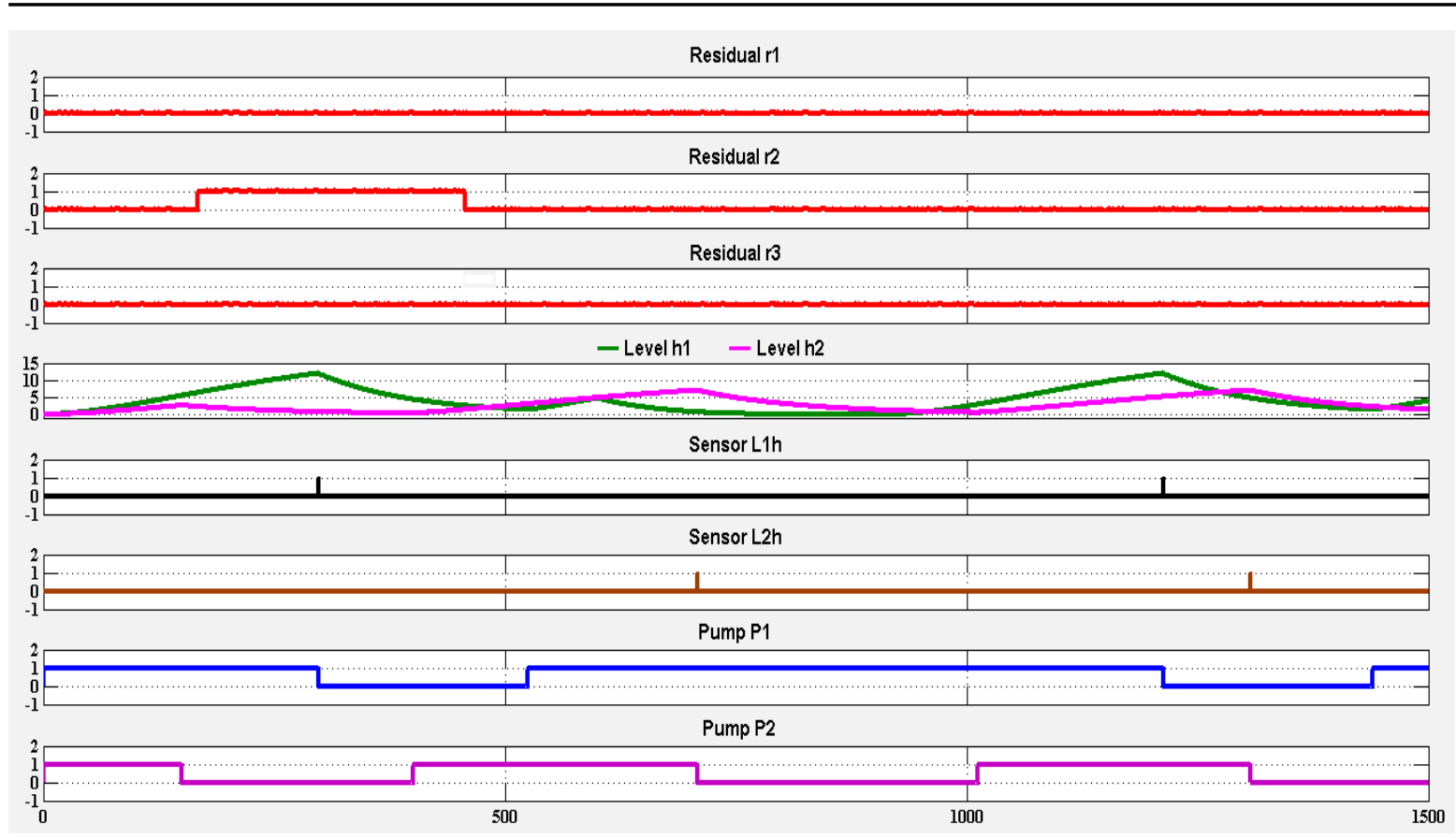

Figure 7. Diagnosis of faults on pump $P_{2}\left(P_{2}\right.$ stuck close $)$ in the presence of disturbances.

$$
\text { where } h_{i}=\frac{q_{i}}{2 g s_{p}^{2}}, i=1,2
$$

$h_{1}$ and $h_{2}$ in a fixed intervals. The TA representing the system of the discrete part in normal operation is given in Figure 6.

The diagnoser construction is based essentially on the temporal knowledge of the system, it is necessary to know the time instant of the pumps $P_{1}$ and $P_{2}$ setting on or the time between the two sensor states. The times of transition determined for each phase of system normal mode are given in Table 2.

\section{Simulation Results}

The water distribution network process is affected by two unknown inputs: a leakage $f(t)$ in the supply pipe of the tank 1 and unknown infiltration $d(t)$ both between $t=600 \mathrm{~s}$ and $t=900 \mathrm{~s}$. As before, the measurements are affected by sensor noises. The diagnosis method proposed in this paper has been validated. Indeed, for each random injection of defects (sensors, actuator or system), the diagnosis approach is able to detect and to isolate faults in the presence of disturbances at all times. We have injected a fault on $P_{2}\left(P_{2}\right.$ stuck_close) so that it remains off at a running request between instants $t=150 \mathrm{~s}$ and $t=400 \mathrm{~s}$ in the presence of the disturbances $f(t)$ and $d(t)$. The responses of the residues $\left(r_{1}, r_{2}, r_{3}\right)$ to this defect are illustrated in Figure 7. The instant of fault detection corresponds to that when $r_{2}$ exceeds its threshold $\varepsilon_{2}\left(\varepsilon_{2}=2 \cdot 10^{-4}\right)$. This instant, called $\mathrm{T}_{\text {detection }}$, is equal to $167.09 \mathrm{~s}$. We note that the signature of $P_{2}$, Table 1 , is confused with those of $Q_{2}$, Tank 3, $P_{l}, L_{1 h}, L_{1 b}, L_{2 h}, L_{2 b}$. Therefore, this defect is only detectable but not locatable. The black and brown signals show the state of the sensors $L_{l h}$ and $L_{2 h}$ and their sensitivity to this defect ( $P_{2}$ stuck_close). The states of both pumps are given by the blue and purple signals. The levels of the liquid, $h_{1}$ in green and $h_{2}$ in rose, are given in Figure 7. In this Figure, we note that $L_{2 h}$ remains at state 0 , till $348.6 \mathrm{~s}$ after setting on the pump $P_{2}$ and the residue $r_{2}$ at this instant is non-zero. Thus, this instant, called $\mathrm{T}_{\text {localization }}$, corresponds to the location of the fault on $P_{2}$. Therefore, this defect is detectable and locatable. Thus, at time $t=600 \mathrm{~s}(f(t) \neq 0$ and $d(t)$ $\neq 0$ ), we note that $L_{l h}$ remains at state 0 , till $297.2 \mathrm{~s}$ after setting on the pump $P_{l}$ and the residues $r_{l}$, $r_{2}$ and $r_{3}$ at the instant $T=897.2 \mathrm{~s}(600 \mathrm{~s}+297.2 \mathrm{~s})$ remain at zero. So this instant represent the instant of the perturbations due to the leakage $f(t)$ and the infiltration $d(t)$ and not a system fault. 


\section{Conclusion}

In this paper we have dealt with the diagnosis of Hybrid Dynamical System (HDS) subject to disturbances. First we have proceeded to HDS modeling by connecting two tools known in the literature. The first is the observer which concerns the continuous part of the HDS and the second is the timed automata (TA) which handles the discrete part. The yielded model is used for diagnosis which is composed of two steps. The first is the fault detection by analyzing the residuals generated by the system output and the observer one. The second step of diagnosis is the fault location which results from signature matrix analyzing and temporal identification of the system. The simulation result raised the advantage of our approach in distinguishing between the defect of a system component and the defect resulting from disturbance.

\section{REFERENCES}

1. Altug, S., Chen, M. Y. \& Trussell, H. J. (1999). Fuzzy inference systems implemented on neural architectures for motor fault detection and diagnosis, IEEE transactions on industrial electronics, 46(6), 1069-1079.

2. Alur, R., Courcoubetis, C., Henzinger, T. A. \& Ho, P. H. (1993). Hybrid automata: An algorithmic approach to the specification and verification of hybrid systems. In Hybrid Systems, LNCS, Vol. 736 (pp. 209-229). Berlin, Heidelberg, Springer.

3. Boutleux, E. (1996). Diagnostic et suivi d'évolution de l'état d'un système par reconnaissance des formes floues: application au modèle $d u$ réseau téléphonique français. Thèse de doctorat Université de Technologie de Compiègne, France.

4. Chow, E. Y. \& Willsky, A. S. (1984). Analytical redundancy and the design of robust failure detection systems, IEEE transaction on Automatic Control, 29(7), 603-614.

5. Ghomri, L. \& Alla, H. (2007). Modeling and analysis using hybrid Petri nets, Nonlinear Analysis: Hybrid Systems, 1(2), 141-153.

6. Kesten, Y. \& Pnueli, A. (1992). Timed and hybrid statecharts and their

textual representation. In International Symposium on Formal Techniques in Real - Time and Fault-Tolerant Systems, LNCS, Vol. 571 (pp. 591-620). Berlin, Heidelberg, Springer.

7. Maaref, B., Simeu-Abazi, Z., Dhouibi, H., Messaoud, H. \& Gascard, E. (2016). Mixed approach for fault diagnosis and fault location of hybrid systems, IFACPapersOnLine, 49(12), 1002-1007.

8. Mehra, R. K. \& Peschon, J (1971). An innovations approach to fault detection and diagnosis in dynamic systems, Automatica, 7(5), 637-640.

9. Ouahi, M. (2011). Observation de systèmes à entrées inconnues, applications à la dynamique automobile. Thèse de doctorat, Université de Limoges.

10. Rajhans, A., Avadhanula, S., Chutinan, A. et al. (2018). Graphical Hybrid Automata with Simulink and Stateflow. In Proceedings of the $21^{\text {st }}$ International Conference on Hybrid Systems: Computation and Control (part of CPS Week) (pp. 267-268). ACM.

11. Sengupta, S., Mukhopadhyay, S., Deb, A., Pattada, K. \& De, S. (2012). Hybrid automata modeling of SI gasoline engines towards state estimation for fault diagnosis, SAE International Journal of Engines, 5(3), 759-781. 
12. Simeu-Abazi, Z., Di Mascolo, M. \& Knotek, M. (2010). Fault diagnosis for discrete event systems: Modelling and verification, Reliability Engineering \& System Safety, 95(4), 369-378.

13. Willsky, A. S. (1976). A survey of design methods for failure detection in dynamic systems, Automatica, 12(6), 601- 611.

14. Xu, J., Loh, A. P. \& Lum, K. Y. (2007). Observer-based fault detection for piecewise linear systems: Continuoustime cases. In IEEE International Conference on Control Applications CCA 2007 (pp. 379-384). Singapore, Singapore, 1 - 3 Oct.

15. Zemoury, M. (2003). Contribution à la surveillance des systèmes de production à l'aide des réseaux de neurones dynamiques: Application à la e-maintenance. Thèse de doctorat, Université de Franche-Comté, France. 